\title{
The Multiplier Effects of Food Relocalization: A Systematic Review
}

\author{
Zsófia Benedek ${ }^{1}\left[\right.$, Imre Fertő ${ }^{1,2, *(\mathbb{C})}$ and Viktória Szente ${ }^{2} \mathbb{D}$ \\ 1 Centre for Economic and Regional Studies, H-1097 Budapest, Hungary; benedek.zsofia@krtk.mta.hu \\ 2 Institute of Marketing and Management, Kaposvár University, H-7400 Kaposvár, Hungary; \\ szente.viktoria@ke.hu \\ * Correspondence: ferto.imre@krtk.mta.hu
}

Received: 23 March 2020; Accepted: 23 April 2020; Published: 25 April 2020

\begin{abstract}
Background: The purchase of local food is often argued to stimulate local economies through multiplier effects; this argument is questioned in this paper. Methods: The ScienceDirect, Wiley Online Library, Taylor and Francis Online, SpringerLink, AgECON, and Emerald databases were searched systematically. A complementary search in Google Scholar was also carried out. Peer-reviewed studies published between 2000 and 2019 were identified using multiple search terms. Data about four types of multipliers (output, employment, income, and value-added multipliers) were extracted. Results: Twenty-four papers fit the criteria, allowing for a qualitative assessment only. Sixteen papers found unambiguously that an increase in demand for local food had a directly positive impact on local economies in some way; one paper found no impact at all. The papers were classified into three groups based on their focal areas: marketing channel-focused, farm enterprise-focused, and scenario/impact analysis-focused studies. In terms of the methods of analysis, three major approaches were identified: input-output models, assessments of descriptive statistics, and econometric analyses. Considerations related to the potential overestimation of current approaches are presented. Conclusions: The existence of employment and income multipliers seems to be more evident, while the impacts of output- and value-added multipliers depend more on the actual context. Research gaps are also identified.
\end{abstract}

Keywords: spillover effect; economic benefit; locally produced food; direct marketing; regional development

\section{Introduction}

Consumer interest in relocalized food is undiminished [1,2] for a number of reasons. For example, higher quality, freshness, safety and healthiness are often mentioned as perceived benefits $[3,4]$. The concept of locally sourced food is popular among policymakers, too, as a potential tool for urban regeneration [5], as well as endogenous rural development [6]. Positive effects on local economies are generally thought to be the consequence of multiplier effects [7-13], although this link has been questioned [14-16]. However, in terms of multipliers, most studies are speculative, and their claims are very rarely supported by rigorous analyses. The authors of [17] note that local food systems "seem to allow a higher share of value added to be retained locally, although quantitative evidence of such impacts is poorly documented" (p. 14).

The idea of multipliers is based on the difference between the initial and the total effects of a specific change [18]. The total effect is composed of direct, indirect, and induced effects [8]. Direct effects quantify the value of new outputs and the additional employment and income generated. Indirect effects are the total value of inputs created by the local suppliers of the focal sector (e.g., involving 
machinery, fertilizer, financial services, etc.). Induced effects measure the impact of workers in the direct and input supply sectors who spend their earnings within a region.

There are several ways to measure the effect of changes on local economies (direct, indirect, and induced) [19]. Based on [18], four types of multipliers are distinguished in this paper. Output multiplier: This measures the change in local sales specified as an increase in demand equivalent to one monetary unit (e.g., 1 USD) for the output of a certain sector; in our case, the increase in demand for relocalized food. In other words, the effect of a rise in demand on the output of all local industries is estimated. Employment multiplier: This measures the new employment which is created to help meet the increased demand (e.g., 1 USD) for the output of the focal sector (relocalized food). This is often expressed in full-time equivalents (FTEs). Income multiplier: This measures the overall change in income within the local economy, received by the employees of a given industry in the case of an increase in demand (1 USD) for relocalized food. Value added multiplier: Here, the performance of a sector is measured in terms of value added, instead of total output (the total value added of all businesses in a state equals the gross national product). For another classification of multiplier effects, see [20].

Multipliers are often calculated from input-output (I-O) tables (transaction matrices) [8], which are representations of national or regional economic accounting that show the flows of products and services between sectors or industries [21]. Knowing how much money is spent on inputs locally (i.e., how money flows from one local industry to another), the cumulative effects of a unit of money on the local economy due to respending can be calculated; see [22]. Social Accounting Matrices (SAMs), an alternative to I-O matrices, focus on different institutions, and are more comprehensive in the sense that, besides economic data, social data (e.g., government welfare payments) are also accounted for [23-25] (another alternative is Computable General Equilibrium models (CGE models)—for a discussion, see, for example, [26-28]). Due to the method of quantification, multiplier effects are typical of localities and also of specific time periods.

Rossi, Johnson and Hendrickson [19] reviewed and classified quantitative studies that focus on the economic impact of local food, based on whether they define local food on the basis of the marketing channel or specified distances; additionally the authors identified whether they use survey-based data about producers and/or consumers or producer databases, and modeled intersectoral relationships to reflect local features. The third system of classification involves defining whether displacement effects are taken into account. Distinguishing between marketing-focused Short Food Supply Chains (SFSCs) and geographically defined Local Food Systems (LFSs) is typical in the related literature $[8,12,17,29]$. Kneafsey, Venn and Schmutz et al. [17] proposed the use of SFSCs, as this concept, while able to incorporate many elements of local food systems, tackles consumer-producer relationships, which are what matter from a community development perspective, without becoming entangled in the different approaches to defining "local." However, Rossi, Johnson and Hendrickson [19] stressed that a marketing channel-based focus might distort results when multipliers are examined as locally produced, but conventionally marketed food is not accounted for, while non-local but directly sold food is.

The concept of multipliers, defined as vehicles of economic growth in general, is often criticized because displaced economic activities (e.g., a decrease in demand for products sold in grocery stores, employment shifting to on-farm work from other industries, etc.) should also be accounted for, reducing the related benefits $[16,19,30,31]$. However, O'Hara and Pirog [32] concluded, in their review, that several studies that accounted for displacement effects were able to identify a positive economic impact at a regional level. DuPuis and Goodman [14] raised the point that the extent of multiplier effects depends on how "locality" is defined; beyond a certain distance, no net effect will be detectable. Deller, Lamie and Stickel [16] reviewed the critiques of multiplier effects in a local food context, noting that the majority of studies are speculative. This criticism is based on the fact that most papers fail to account for displacement effects, that some studies de facto find weak or no impacts, even in the case of positive economic outcomes, and because uncertainty exists with respect to scalability. 
A systematic literature review was conducted to clarify the nature of multiplier effects related to relocalized food. Accordingly, the aim of the paper is threefold, with the first aim being to identify whether food relocalization (i.e., an increase in the consumption of local food by members of civil society) generally has multiplier effects. Finding that multiplier effects are generally believed to exist, we reveal the typical mechanisms (i.e., which types of multiplier effect) that prevail. From a methodological perspective, approaches to the quantitative study of multipliers are also addressed.

Following the suggestion of [17] and the perspective of [33], a participatory, community-development-centered approach was applied during the formulation of the research question. Although the validity of the argument of [19] that the concept of LFS can produce better estimates of economic impact is recognized, we believe that the need for further specification of "local" (i.e., the geographical distance that should be taken into account), makes a broad territorial synthesis impossible in light of the relatively small number of studies in the sample. Also, when the development of LFSs is encouraged, producers involved in close consumer-producer relationships (and organizers working in specific marketing channels) can be addressed, so, from a policy (economic development) perspective, relationships matter [16,17,33]. Furthermore, as Rossi, Johnson and Hendrickson [19] also noted, the growth of the local food sector has already been foreseen (in relation to that of conventional food systems), irrespective of the actual locus of production. In this paper, the terms "local food" and "relocalized food" are used interchangeably. The purpose of using the term "relocalized" is to suggest that the relatively new local food movement is a more typical focus of wealthier economies [17,34,35]; accordingly, an appraisal of the urban-rural agricultural linkages of the global south (an approach adopted by [36-38] and others) is not the focus of this investigation.

Any systematic review, as opposed to a traditional descriptive literature review, requires a clearly formulated research question, an extensive literature search that identifies all potentially interesting primary sources, transparent study inclusion and exclusion criteria, synthesis of data, and interpretation of results [39]. This well-defined and rigorous process helps eliminate biases and permits the generalization of outcomes across populations and in specific circumstances [40]. Systematic reviews are in demand in evidence-based policymaking [41,42].

The present study contributes to the literature in several ways. Firstly, it synthesizes opposing findings related to the multiplier effects of relocalized food. Secondly, being a systematic review that includes only peer-reviewed papers (see the following section), study selection is transparent and findings rigorous. Thirdly, different types of multipliers are specifically and exhaustively addressed.

\section{Materials and Methods}

In this paper, a process that follows the Preferred Reporting Items for Systematic Reviews and Meta-Analyses (PRISMA) guidelines [40,43] is introduced. PRISMA, although originally developed in a healthcare context, is a popular and rigorous guideline that is also used in the field of agricultural economics [2,44-46], among other areas, when analyzing and reporting on search-related results. To establish greater scientific rigor, the protocol for the present systematic review process was discussed at the 174th Seminar of the European Association of Agricultural Economists in Matera, Italy in October 2019.

The following electronic databases were reviewed for papers: ScienceDirect, Wiley Online Library, Taylor and Francis Online, SpringerLink, AgECON, and Emerald and, following the approach of [2,45], a complementary search in Google Scholar was also carried out. All the studies that are referred to here are original research papers that were published in English, either in print or electronic form, between January 2000 and December 2019. Due to the focus on a participatory approach, economic impact studies of the institutional purchasing of local food (e.g., studies that focus on farm-to-school programs) were excluded. 
The previous section described the problems related to the definition of local food systems and short food supply chains. To remain as inclusive as possible, a broad selection of related keywords was defined (see Table 1). Thus, following the approach of [2] and others, we accept that papers use their own definitions of local (relocalized) food. All the combinations of keywords defined under Concept 1 and Concept 2 were used, resulting in 42 searches in each database (a total of 252 searches in all databases).

Table 1. Search strings.

\begin{tabular}{cc}
\hline Concept 1 & Concept 2 \\
\hline "multiplier effect" & "local food" \\
"economic benefit" & "locally produced" \\
"regional development" & "locally grown" \\
"local economy" & "alternative food network" \\
"regional economy" & "short food supply chain" \\
& "alternative food system" \\
& "alternative agri-food network" \\
\hline
\end{tabular}

The number of keywords and combinations is relatively high compared to other systematic reviews in the field of agricultural economics (e.g., [2,47,48]). This is because both of our key concepts ("multiplier effect" and "local food") have multiple synonyms.

After the database search, duplicates were eliminated and the remaining titles were screened. In a further step, all the abstracts of the 655 papers with promising titles were read to compile a pool of papers for full text screening $(n=116)$. Although we searched for original estimations of the multiplier effect of food relocalization, reviews were included to identify whether further eligible papers could be found based on their reference lists. To reduce bias, full texts were screened and data were extracted by two independent reviewers. When discrepancies arose, a third reviewer was involved. The reference lists of the identified papers were also checked for further eligible papers. Thirteen papers were identified through the systematic database searches, two $([49,50])$ were found in Google Scholar, and nine were found through the screening of the reference lists. Thus, a total of 24 papers were included in the analysis. Figure 1 illustrates the research process with the number of papers identified at each step of the research process.

The following pieces of information were extracted from all the identified papers $(n=24)$ : subject (as the focus of the study); year(s) of study, method(s) of data collection and sampling; method(s) of analysis; location; sample size; key findings; reliability (whether biases were mentioned or discussed); validity (whether discussed). 


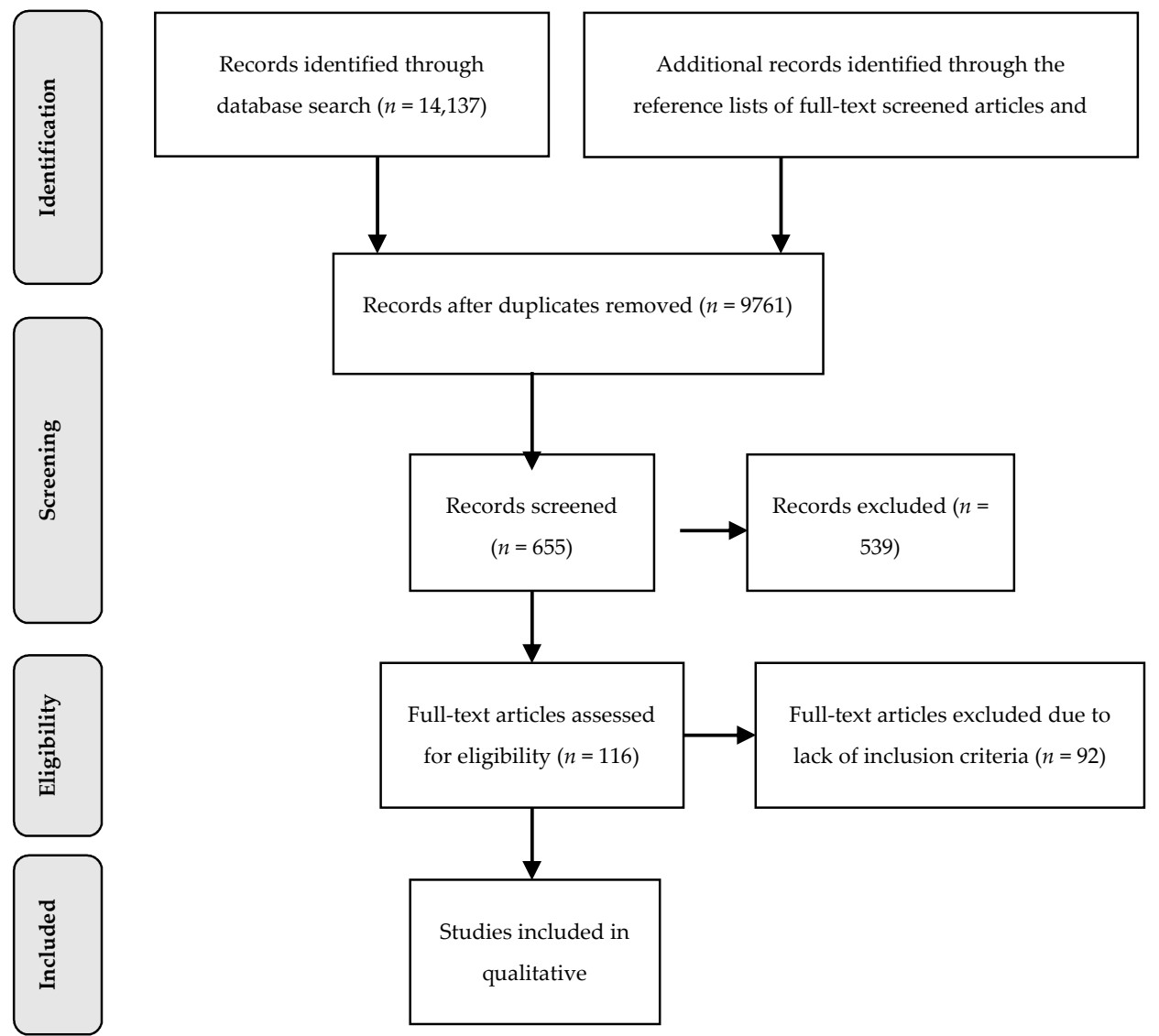

Figure 1. Overview of research process, including number of papers identified at different stages (the Preferred Reporting Items for Systematic Reviews and Meta-Analyses (PRISMA) flow diagram that is distributed under the terms of the Creative Commons Attribution License, see [40]).

\section{Results and discussion}

A summary of the 24 papers is shown in Table 2. Studies are classified first based on their subject of study, followed by the method of analysis; finally, they are ordered chronologically. Papers in which negative or partially negative results are published are indicated in bold text (negative results are understood as meaning no multiplier effect was found, or that the multiplier effect found in the local food context was less than that of conventional production). Due to the low number and heterogeneity of the applied methods, qualitative assessments were included, in a similar manner to the syntheses prepared by $[45,48,51]$, although there were fewer papers involved in the analysis in our case.

The majority of studies address the situation in English-speaking territories (22/24). Out of the 24 sample papers, 17 are connected to the USA alone, while a further one includes a USA-Ontario (Canada) comparison (the latter area is mostly English-speaking). A similar pattern was identified by other systematic reviews that focused on different aspects of local food; see, for example, [2,48,51,52]. One paper evaluates the situation in Australia, two focus on the UK alone, and one in comparison with Poland. As for non-English-speaking territories, one paper describes the situation in the French part of Canada, and one the Czech Republic (in addition to the above-mentioned Polish-British comparison).

The logic of the assessment is as follows (similar to the layout of Table 2). First, studies are classified based on the subject of the study. Then, the focus is on the method of analysis. Finally, the types of multipliers are considered in order to assess the existence of economic impacts. 
Table 2. Key findings.

\begin{tabular}{|c|c|c|c|c|c|c|c|c|c|}
\hline Reference & Subject & Year(s) of Study & $\begin{array}{l}\text { Methods (Data, } \\
\text { Sampling) }\end{array}$ & Methods (Analysis) & Location & Sample & Key Findings & Reliability (Biases) & Validity \\
\hline [53] & $\begin{array}{l}\text { Farmers' markets } \\
\text { (randomly selected) }\end{array}$ & 2002 & $\begin{array}{l}\text { Questionnaire (FM } \\
\text { managers, vendors, } \\
\text { consumers); } \\
\text { IMPLAN-based data. }\end{array}$ & $\begin{array}{l}\text { IMPLAN-based } \\
\text { input-output model }\end{array}$ & $\begin{array}{l}\text { Oklahoma } \\
\text { (USA) }\end{array}$ & $\begin{array}{c}n=21 \mathrm{FM} \\
\text { managers, } n=64 \\
\text { vendors, } n=312 \\
\text { consumers }\end{array}$ & $\begin{array}{l}\text { Output- and value-added multipliers. } \\
\text { Famers' markets generate total direct } \\
\text { sales of } \$ 3.3 \text { million, with total economic } \\
\text { impact of almost } \$ 6 \text { million. } \\
\text { Output and employment multipliers. } 69\end{array}$ & Not discussed & Not discussed \\
\hline [31] & Farmers' markets & 2005 & $\begin{array}{l}\text { Questionnaire } \\
\text { (vendors); } \\
\text { IMPLAN-based data }\end{array}$ & $\begin{array}{c}\text { IMPLAN-based } \\
\text { input-output model }\end{array}$ & $\begin{array}{l}\text { West Virginia } \\
\text { (USA) }\end{array}$ & $n=183$ vendors & $\begin{array}{l}\text { FTE jobs, } \$ 2389 \mathrm{M} \text { in output }(\$ 1.48 \mathrm{M} \\
\text { GSP). If direct revenue losses are } \\
\text { included (groceries), the impact is } \\
\text { reduced: } 43 \text { FTE jobs, } \$ 1075 \mathrm{M} \text { in output } \\
\text { (\$0.653 M in GSP). }\end{array}$ & $\begin{array}{l}\text { Reliability of } \\
\text { instrument was } \\
\text { tested (Cronbach's } \\
\text { alpha). }\end{array}$ & $\begin{array}{l}\text { Survey questions } \\
\text { were tested with } \\
\text { four volunteer } \\
\text { vendors. }\end{array}$ \\
\hline [54] & $\begin{array}{l}\text { Farmers' markets and } \\
\text { impact analysis of a } \\
\text { state-level locally } \\
\text { grown campaign }\end{array}$ & 2011 & $\begin{array}{l}\text { Two stratified surveys } \\
\text { of FMs (managers and } \\
\text { vendors); a random } \\
\text { mail survey of } \\
\text { consumers. }\end{array}$ & $\begin{array}{l}\text { IMPLAN-based } \\
\text { Social Accounting } \\
\text { Matrix (SAM) model }\end{array}$ & $\begin{array}{l}\text { South } \\
\text { Carolina } \\
\text { (USA) }\end{array}$ & $\begin{array}{c}n=12 \mathrm{FM} \\
\text { managers, number } \\
\text { of vendors not } \\
\text { reported, } n=165 \\
\text { consumers }\end{array}$ & $\begin{array}{l}\text { Output multiplier, no value-added } \\
\text { multiplier. Total sales at FMs are } \\
\text { estimated to be } \$ 7.533 \text { million. The } \\
\text { campaign (increased sales at FMs) } \\
\text { apparently did not make a major } \\
\text { contribution to the state economy. }\end{array}$ & Not discussed & Not discussed \\
\hline [55] & $\begin{array}{l}\text { Two specific farmers' } \\
\text { markets (Kenosha } \\
\text { Harbor Market, } \\
\text { Waukesha FM) }\end{array}$ & 2008 & $\begin{array}{l}\text { Questionnaire (vendors, } \\
\text { customers, } \\
\text { storeowners); } \\
\text { semi-structured } \\
\text { interviews. }\end{array}$ & $\begin{array}{c}\text { Assessment of } \\
\text { descriptive statistics }\end{array}$ & $\begin{array}{c}\text { Kenosha, } \\
\text { Waukesha } \\
\text { (Wisconsin } \\
\text { USA) }\end{array}$ & $\begin{array}{c}n=96 \text { vendors, } n= \\
575 \text { customers, } n= \\
54 \text { storeowners }\end{array}$ & $\begin{array}{l}\text { Output multiplier. Direct economic } \\
\text { benefit from FMs to downtown } \\
\text { storeowners: } \$ 637,485 \text { (Kenosha); } \\
\$ 340,812 \text { (Waukesha). Indirect economic } \\
\text { benefits are also acknowledged. }\end{array}$ & Not discussed & Not discussed \\
\hline [56] & $\begin{array}{l}\text { Three specific farmers' } \\
\text { markets (Flint Farmers' } \\
\text { Market; Western Fair } \\
\text { Farmers' and Artisans' } \\
\text { Market in London) }\end{array}$ & 2011 & $\begin{array}{l}\text { Consumer survey, no. } \\
\text { customers, interviews } \\
\text { with market managers. }\end{array}$ & $\begin{array}{l}\text { Extrapolation of } \\
\text { average consumer } \\
\text { spending. } \\
\text { Market-specific } \\
\text { multipliers were } \\
\text { created following the } \\
\text { I-O-based Econsult } \\
\text { Corporation (2007). }\end{array}$ & $\begin{array}{l}\text { Flint } \\
\text { (Michigan, } \\
\text { USA) London } \\
\text { (Ontario, } \\
\text { Canada) }\end{array}$ & $\begin{array}{l}n=405 \text { consumers } \\
\text { in Flint, } n=490 \\
\text { consumers in } \\
\text { London. }\end{array}$ & $\begin{array}{l}\text { Value added multiplier for London: } 1.47 \text {; } \\
\text { Flint: 1.39. The annual impact of the } \\
\text { London farmers' market is CA } \$ 7.0 \mathrm{M} \text {, } \\
\text { while the impact of the Flint farmers' } \\
\text { market is US } \$ 6.8 \mathrm{M} \text { annually. }\end{array}$ & Not discussed & $\begin{array}{c}\text { The benchmark } \\
\text { approach was } \\
\text { validated. }\end{array}$ \\
\hline [57] & Farmers' markets & 2011 & $\begin{array}{l}\text { Interviews; focus group } \\
\text { discussions }\end{array}$ & $\begin{array}{l}\text { Qualitative case } \\
\text { study }\end{array}$ & $\begin{array}{c}\text { Czech } \\
\text { Republic }\end{array}$ & $n=35 \mathrm{FM}$ managers & $\begin{array}{l}\text { No multiplier. The effect of FMs on } \\
\text { rural areas in Czechia is very limited } \\
\text { and is not expected to expand further. } \\
\text { The benefits of these markets lie in } \\
\text { non-economic values. }\end{array}$ & Not discussed & Not discussed \\
\hline [58] & SFSCs & 2012 & $\begin{array}{l}\text { Questionnaire } \\
\text { (households); } \\
\text { IMPLAN-based data }\end{array}$ & $\begin{array}{l}\text { IMPLAN-based } \\
\text { input-output model }\end{array}$ & Florida (USA) & $\begin{array}{c}n=1599 \\
\text { households }\end{array}$ & $\begin{array}{l}\text { Output, employment, income and } \\
\text { value-added multipliers are estimated. } \\
\$ 19.20 \text { billion in industry output, } \$ 851 \\
\text { million in indirect business taxes; } \\
183,625 \text { full-time and part-time jobs; } \\
\$ 6.46 \text { billion in labor income; } \$ 10.47 \\
\text { billion in value-added, including } \\
\text { regional multiplier effects for } \\
\text { agricultural production and wholesale } \\
\text { and retail distribution (2013 prices). }\end{array}$ & $\begin{array}{l}\text { Response bias and } \\
\text { social desirability } \\
\text { bias are discussed. }\end{array}$ & Not discussed \\
\hline [59] & SFSCs & 2010 & $\begin{array}{l}\text { Questionnaire; } \\
\text { interviews; price } \\
\text { survey (in grocery } \\
\text { stores, on farm and on } \\
\text { the online platform of } \\
\text { local food hubs) }\end{array}$ & $\begin{array}{c}\text { Assessment of } \\
\text { descriptive statistics }\end{array}$ & $\begin{array}{l}\text { Lac-Saint-Jean-Es } \\
\text { Lotbiniére, } \\
\text { Brome-Missisquo } \\
\text { (Quebec, } \\
\text { Canada) }\end{array}$ & 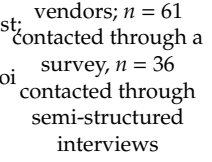 & $\begin{array}{l}\text { Employment multiplier. SFSC farms } \\
\text { create on average four FTE jobs per farm } \\
(0.75 \text { per ha) compared to } 2.5 \text { ( } 0.19) \text { for } \\
\text { the Quebec average. }\end{array}$ & Not discussed & $\begin{array}{l}\text { Limitations are } \\
\text { discussed }\end{array}$ \\
\hline
\end{tabular}


Table 2. Cont

\begin{tabular}{|c|c|c|c|c|c|c|c|c|c|}
\hline Reference & Subject & Year(s) of Study & $\begin{array}{l}\text { Methods (Data, } \\
\text { Sampling) }\end{array}$ & Methods (Analysis) & Location & Sample & Key Findings & Reliability (Biases) & Validity \\
\hline [60] & Local food system & 2011 & IMPLAN data & $\begin{array}{l}\text { IMPLAN-based } \\
\text { input-output model. } \\
\text { A model was } \\
\text { constructed to } \\
\text { account for local food } \\
\text { systems as a sector }\end{array}$ & $\begin{array}{l}\text { Michigan } \\
\text { (USA) }\end{array}$ & & $\begin{array}{l}\text { Output, employment and income } \\
\text { multipliers. The local food system } \\
\text { generated } \$ 4.53 \text { billion in total output in } \\
\text { Michigan, supporting } 18,627 \text { jobs with } \\
\text { total earnings of } \$ 680.5 \text { million. }\end{array}$ & $\begin{array}{c}\text { Several potential } \\
\text { biases are discussed }\end{array}$ & Not discussed \\
\hline [61] & Local food system & 2013 & IMPLAN data & $\begin{array}{l}\text { IMPLAN-based } \\
\text { input-output model. } \\
\text { A model was } \\
\text { constructed to } \\
\text { account for local food } \\
\text { systems as a sector. } \\
\text { IMPLAN-based }\end{array}$ & Idaho (USA) & & $\begin{array}{l}\text { Output multiplier. Six models (three } \\
\text { local food definitions, two scenarios) are } \\
\text { presented. Positive output effects of } \\
\text { wide range }(364 \text { million-27.2 billion } \\
\text { dollars) estimated. }\end{array}$ & $\begin{array}{l}\text { Several limitations } \\
\text { (potential biases) } \\
\text { are discussed }\end{array}$ & Not discussed \\
\hline [62] & $\begin{array}{l}\text { "Regional Access" (RA) } \\
\text { local food hub, and } \\
\text { farms selling products } \\
\text { for RA. }\end{array}$ & 2011 & $\begin{array}{l}\text { Interviews (food hub } \\
\text { personnel, farm } \\
\text { operators); an online } \\
\text { customer survey; } \\
\text { IMPLAN-based data }\end{array}$ & $\begin{array}{l}\text { input-output model. } \\
\text { The effect of } \\
\text { hypothetical shocks } \\
\text { (\$1 million increase in } \\
\text { final demand) are } \\
\text { modelled when } \\
\text { negative spending in } \\
\text { other sectors is also } \\
\text { considered. }\end{array}$ & $\begin{array}{l}\text { New York } \\
\text { (USA) }\end{array}$ & $\begin{array}{c}n=1 \text { food hub; } n= \\
30 \text { farms }(35 \%) n= \\
305 \text { customers }\end{array}$ & $\begin{array}{l}\text { Output and employment multipliers. } \\
\text { Output multiplier: } 1.75 \text {; employment } \\
\text { multiplier: } 2.14 \text {. Based on customer } \\
\text { surveys, every } \$ 1 \text { increase in final } \\
\text { demand for food hub products } \\
\text { generates a } \$ 0.11 \text { reduction in purchases } \\
\text { in other sectors. }\end{array}$ & $\begin{array}{l}\text { The downward bias } \\
\text { associated with } \\
\text { using default } \\
\text { agricultural } \\
\text { production data of } \\
\text { farms is discussed. }\end{array}$ & Not discussed \\
\hline [49] & $\begin{array}{l}\text { An online retailer of } \\
\text { locally sourced food } \\
\text { and drink (edibLE16) }\end{array}$ & 2014-2017 & $\begin{array}{l}\text { Empirical financial data } \\
\text { collected over a } \\
\text { three-year period; } \\
\text { interviews with } \\
\text { suppliers of edibLE16 } \\
\text { (second round), and } \\
\text { with their suppliers } \\
\text { (third round) }\end{array}$ & $\begin{array}{l}\text { LM3 method: Local } \\
\text { Multiplier to the third } \\
\text { round }\end{array}$ & $\begin{array}{l}\text { Market } \\
\text { Harborough } \\
\text { (UK) }\end{array}$ & $\begin{array}{l}n=1 \text { online retailer; } \\
n=21 \text { second round } \\
\text { upstream suppliers }\end{array}$ & $\begin{array}{l}\text { Output multiplier. An initial } £ 1.00 \\
\text { investment with edibLE16 generated } \\
\text { between } £ 0.95 \text { and } £ 1.24 \text { of additional } \\
\text { sales, depending on method applied. }\end{array}$ & $\begin{array}{l}\text { The limitations of } \\
\text { the LM3 approach } \\
\text { and the reliability of } \\
\text { the results are } \\
\text { discussed. }\end{array}$ & Not discussed \\
\hline [63] & Local craft breweries & 2014 & $\begin{array}{l}\text { Questionnaire; } \\
\text { semi-structured } \\
\text { interviews }\end{array}$ & $\begin{array}{c}\text { Assessment of } \\
\text { descriptive statistics }\end{array}$ & $\begin{array}{l}\text { New South } \\
\text { Wales and } \\
\text { Tasmania } \\
\text { (Australia) }\end{array}$ & $n=16$ brewers & $\begin{array}{l}\text { Employment multiplier (expansion of } \\
\text { FTE employment). }\end{array}$ & Not discussed & Not discussed \\
\hline [64] & $\begin{array}{l}\text { Farm enterprises (a } \\
\text { comparison of two } \\
\text { "local" dairy farms } \\
\text { characterized by } \\
\text { different types of } \\
\text { production) }\end{array}$ & 2014 & $\begin{array}{l}\text { Interviews with key } \\
\text { stakeholders. } \\
\text { Secondary data } \\
\text { obtained from official } \\
\text { government websites }\end{array}$ & $\begin{array}{l}\text { Assessment of } \\
\text { descriptive statistics } \\
\text { related to operational } \\
\text { costs }\end{array}$ & $\begin{array}{l}\text { Two Hawaiian } \\
\text { islands } \\
\text { (Kaua'i and } \\
\text { Hawai'i), USA }\end{array}$ & $n=2$ farms & $\begin{array}{l}\text { No output multiplier, income } \\
\text { multiplier. } 17 \text { and } 19 \% \text { of expenses went } \\
\text { on labor. The majority ( } 73 \% \text { and } 59 \%) \\
\text { of expenditure went to non-local } \\
\text { inputs (no output multiplier). }\end{array}$ & Not discussed & Not discussed \\
\hline [19] & Farm enterprises & 2012 & $\begin{array}{l}\text { Questionnaire } \\
\text { (producers); } \\
\text { IMPLAN-based data }\end{array}$ & $\begin{array}{l}\text { IMPLAN-based } \\
\text { input-output model }\end{array}$ & $\begin{array}{l}\text { Old Trails and } \\
\text { North Ozark } \\
\text { regions, } \\
\text { Missouri; } \\
\text { Southeast } \\
\text { Nebraska } \\
\text { (USA) }\end{array}$ & $\begin{array}{c}n=32 \text { local } \\
\text { producers involved } \\
\text { in } \\
\text { direct-to-consumer } \\
\text { sales }\end{array}$ & $\begin{array}{l}\text { Output and value added multipliers } \\
\text { depending on the context; employment } \\
\text { multiplier. OM: } 1.53-1.77 \text {; EM: } 1.05-1.08 \text {; } \\
\text { VAM: } 1.92-2.5 \text {. In two out of the three } \\
\text { studied cases local food sales } \\
\text { generated more total regional sales, } \\
\text { and GDP than conventional sales. The } \\
\text { employment effect of local food was } \\
\text { higher everywhere. }\end{array}$ & Not discussed & Not discussed \\
\hline
\end{tabular}


Table 2. Cont

\begin{tabular}{|c|c|c|c|c|c|c|c|c|c|}
\hline Reference & Subject & Year(s) of Study & $\begin{array}{l}\text { Methods (Data, } \\
\text { Sampling) }\end{array}$ & Methods (Analysis) & Location & Sample & Key Findings & Reliability (Biases) & Validity \\
\hline [50] & Farm enterprises & 2014 & $\begin{array}{l}\text { Interviews (farm } \\
\text { operators); } \\
\text { IMPLAN-based data }\end{array}$ & $\begin{array}{l}\text { IMPLAN-based } \\
\text { input-output model; } \\
\text { another production } \\
\text { function was } \\
\text { constructed from } \\
\text { detailed farm } \\
\text { financial data. } \\
\text { Substitution effect } \\
\text { was accounted for. }\end{array}$ & $\begin{array}{l}\text { Minnesota } \\
\text { (USA) }\end{array}$ & $n=11$ & $\begin{array}{l}\text { Output, employment and income } \\
\text { multipliers. OM: } 1.4-1.6 ; \text { EM: } 9-100 \text { jobs; } \\
\text { IM: US } \$ 1 \text { million in sales will support } \\
\text { US } \$ 506,600-568,600 \text { of labor income. } \\
\text { The constructed production function } \\
\text { predicted a higher impact than the } \\
\text { IMPLAN model. Farms involved in } \\
\text { local food systems might have a greater } \\
\text { positive impact than direct-to-wholesale } \\
\text { enterprises. }\end{array}$ & Not discussed & Not discussed \\
\hline [65] & $\begin{array}{c}\text { Farm enterprises } \\
\text { (association between } \\
\text { community-focused } \\
\text { agriculture and growth } \\
\text { in total agricultural } \\
\text { sales) }\end{array}$ & $\begin{array}{l}\text { Change: } 2002 \\
-2007\end{array}$ & $\begin{array}{l}\text { Published sources } \\
\text { (Census of Agriculture } \\
\text { data) }\end{array}$ & $\begin{array}{l}\text { Econometric analysis } \\
\text { (IV regressions) }\end{array}$ & US counties & $n=3078$ & $\begin{array}{l}\text { Income multiplier, no value-added } \\
\text { multiplier. A } \$ 1 \text { increase in farm sales } \\
\text { led to an annualized increase of } \$ 0.04 \text { in } \\
\text { county-level personal income. } \\
\text { Community-focused agriculture did not } \\
\text { make a significant contribution to } \\
\text { economic growth. }\end{array}$ & $\begin{array}{l}\text { Potential for } \\
\text { endogeneity bias, } \\
\text { IV regression is } \\
\text { undertaken }\end{array}$ & $\begin{array}{l}\text { Strength, validity, } \\
\text { and necessity of } \\
\text { using the IV mode } \\
\text { were tested. }\end{array}$ \\
\hline [66] & Farm enterprises & 2004 & $\begin{array}{l}\text { USDA Agricultural } \\
\text { Resource Management } \\
\text { Survey; secondary } \\
\text { county data }\end{array}$ & $\begin{array}{l}\text { Econometric analysis } \\
\text { (multinomial logit } \\
\text { models) }\end{array}$ & 48 US states & $\begin{array}{l}\text { Representative } \\
\text { national sample of } \\
\text { farm households }\end{array}$ & $\begin{array}{l}\text { No output multiplier, income } \\
\text { multiplier. Urban area farms: } 20-25 \% \text { of } \\
\text { farm business expenses went on labor; } \\
\text { household goods are bought in closest } \\
\text { markets, farm business items are } \\
\text { purchased further away. Rural } \\
\text { locations-opposite pattern. Most } \\
\text { remote counties: labor costs were only } \\
\text { 6-7\% of total expenditures; a large } \\
\text { proportion of budget may be spent } \\
\text { outside the local community (no } \\
\text { output multiplier). }\end{array}$ & Not discussed & $\begin{array}{l}\text { One of the model } \\
\text { details was } \\
\text { validated }\end{array}$ \\
\hline [67] & Farm enterprises & 2008 & $\begin{array}{l}\text { Questionnaire; } \\
\text { telephone-based } \\
\text { (England); face-to-face } \\
\text { survey (Poland). }\end{array}$ & $\begin{array}{l}\text { Econometric analysis } \\
\text { (multivariate probit } \\
\text { analysis) }\end{array}$ & $\begin{array}{c}\text { Podlaskie } \\
\text { (Poland); } \\
\text { North East } \\
\text { Scotland (UK) }\end{array}$ & $\begin{array}{l}n=224(\mathrm{UK}) ; n= \\
244 \text { (Poland). } 60 \% \\
\text { cattle breeding } \\
\text { farms (UK); } 50 \% \\
\text { dairy farms } \\
\text { (Poland) }\end{array}$ & $\begin{array}{l}\text { Output multiplier depending on the } \\
\text { context. Local farm transaction in North } \\
\text { East Scotland covers a far larger area } \\
\text { than in Podlaskie. Transactions have } \\
\text { multiple centers (specific towns) in UK, } \\
\text { while spatially diffuse in Poland. }\end{array}$ & Not discussed & $\begin{array}{l}\text { Representativeness } \\
\text { of the sample and } \\
\text { further limitations } \\
\text { are discussed. }\end{array}$ \\
\hline [68] & $\begin{array}{l}\text { Organic and } \\
\text { non-organic farm } \\
\text { enterprises }\end{array}$ & 2004 & $\begin{array}{l}\text { Questionnaire; the } \\
\text { original sample ( } \mathrm{n}= \\
1684 ; 684 \text { organic) was } \\
\text { from Defra's census } \\
\text { branch and was } \\
\text { stratified. }\end{array}$ & $\begin{array}{c}\text { Assessment of } \\
\text { descriptive statistics }\end{array}$ & England (UK) & $\begin{array}{c}n=302 \text { organic } \\
\text { farm managers, } n= \\
353 \text { non-organic } \\
\text { farm managers }\end{array}$ & $\begin{array}{l}\text { Employment multiplier. Organic farms } \\
\text { generate more employment (including } \\
\text { greater proportion of non-family FTEs) } \\
\text { and slightly higher salary for non-family } \\
\text { members. }\end{array}$ & Not discussed & Not discussed \\
\hline [69] & $\begin{array}{l}\text { Scenario analysis: } \\
\text { fulfilling dietary } \\
\text { guidelines by eating } \\
\text { more local fresh } \\
\text { produce }\end{array}$ & 2004 & $\begin{array}{l}\text { Multiple published } \\
\text { sources }\end{array}$ & $\begin{array}{l}\text { IMPLAN-based } \\
\text { input-output model }\end{array}$ & $\begin{array}{l}\text { Michigan } \\
\text { (USA) }\end{array}$ & $\begin{array}{l}\text { Representative } \\
\text { sample of Michigan } \\
\text { residents }\end{array}$ & $\begin{array}{l}\text { Employment and income multipliers. } \\
\text { Net increase of } 1780 \text { jobs and a total net } \\
\text { increase of } \$ 211 \text { million in income. }\end{array}$ & Not discussed & Not discussed \\
\hline
\end{tabular}


Table 2. Cont.

\begin{tabular}{|c|c|c|c|c|c|c|c|c|c|}
\hline Reference & Subject & Year(s) of Study & $\begin{array}{l}\text { Methods (Data, } \\
\text { Sampling) }\end{array}$ & Methods (Analysis) & Location & Sample & Key Findings & Reliability (Biases) & Validity \\
\hline$[70]$ & $\begin{array}{l}\text { Scenario analysis: } \\
\text { expected effects of a } \\
\text { potential exogenous } \\
\text { policy shock resulting } \\
\text { in a } \$ 1 \mathrm{M} \text { increase in } \\
\text { final demand for } \\
\text { small-scale direct } \\
\text { agricultural products }\end{array}$ & 2011 & $\begin{array}{l}\text { A mixed-method } \\
\text { approach: survey and } \\
2010 \text { IMPLAN data. }\end{array}$ & $\begin{array}{l}\text { IMPLAN-based } \\
\text { input-output models } \\
\text { with regional } \\
\text { purchase coefficients }\end{array}$ & $\begin{array}{l}\text { New York } \\
\text { (USA) }\end{array}$ & $\begin{array}{c}n=77 \text { farm } \\
\text { managers }\end{array}$ & $\begin{array}{c}\text { No output multiplier, no employment } \\
\text { multiplier, income and value-added } \\
\text { multipliers. SDA sectors have } \\
\text { (pre-shock) annual total output of } \$ 56 \mathrm{M} \text {. } \\
\text { Similar initial shock caused } 3.6 \% \text { lower } \\
\text { output impacts and 16.6\% lower total } \\
\text { employment in the SDA than in the } \\
\text { non-SDA sector. Total effects for labor } \\
\text { income and total value added are } 7.3 \% \\
\text { and } 8.7 \% \text { higher, respectively, for the } \\
\text { SDA sector. }\end{array}$ & Not discussed & Not discussed \\
\hline [71] & $\begin{array}{l}\text { Scenario analysis: two } \\
\text { potential policy tools } \\
\text { for improving local } \\
\text { food production in } \\
\text { Hawaii }\end{array}$ & $\begin{array}{c}2012 \text { was chosen } \\
\text { as a base year }\end{array}$ & $\begin{array}{l}\text { Multiple published } \\
\text { sources }\end{array}$ & $\begin{array}{l}\text { Positive mathematical } \\
\text { programming model. } \\
\text { [Calibrated both on } \\
\text { supply and demand } \\
\text { sides] }\end{array}$ & $\begin{array}{l}\text { Four counties } \\
\text { in Hawaii } \\
\text { (USA) }\end{array}$ & $\begin{array}{l}\text { Representative } \\
\text { sample of Hawaiian } \\
\text { farm businesses } \\
\text { and consumers }\end{array}$ & $\begin{array}{l}\text { Value added multiplier. An economic } \\
\text { gain of } \$ 118 \text { per } \$ 100 \text { invested (as tax } \\
\text { exemption). On the other hand, } \\
\text { investment in } 1200 \text { acres ( } 485 \text { ha) of land } \\
\text { may generate gains of as much as } \$ 357 \\
\text { per } \$ 100 \text { annual investment. }\end{array}$ & $\begin{array}{l}\text { Simulation results } \\
\text { were variously } \\
\text { tested; potential } \\
\text { biases are } \\
\text { discussed. }\end{array}$ & $\begin{array}{l}\text { A detailed } \\
\text { validation } \\
\text { procedure is } \\
\text { documented. }\end{array}$ \\
\hline [72] & $\begin{array}{l}\text { Scenario analysis: } \\
\text { potential food } \\
\text { self-reliance in the City } \\
\text { of Cleveland }\end{array}$ & $\begin{array}{l}\text { No specified time } \\
\text { horizon }\end{array}$ & $\begin{array}{l}\text { Multiple published } \\
\text { sources }\end{array}$ & $\begin{array}{l}\text { Scenario analysis. } \\
\text { Self-reliance is } \\
\text { calculated using a } \\
\text { given formula }\end{array}$ & $\begin{array}{l}\text { Cleveland } \\
\text { (USA) }\end{array}$ & & $\begin{array}{l}\text { Value-added multiplier. Enhanced food } \\
\text { self-reliance would result in } \$ 29 \mathrm{M}-\$ 115 \\
\text { M being retained in Cleveland annually } \\
\text { depending upon the scenario. }\end{array}$ & $\begin{array}{l}\text { Limitations of } \\
\text { selected measures } \\
\text { are discussed. }\end{array}$ & Not discussed \\
\hline
\end{tabular}

Note: papers in which negative or partially negative results are published are indicated in bold text. Abbreviations: full-time equivalent (FTE); instrumental variables (IV); million (M); Note: papers in which negative or partially negative results are published are indicated in bold text. Abbreviations: full-time equivalent (FTE); instrumental variables (IV); million (M);
Impact Analysis for Planning (IMPLAN); farmers' market (FM); Short Food Supply Chain (SFSC); Gross State Product (GSP); Small Direct Agricultural producer (SDA); input-output (I-O). 


\subsection{Subject of Study}

In line with the findings of [19,32], it is remarkable to see how many entirely different perspectives multiplier effects can be studied from. Based on the subject of the studies (i.e., in which context the multiplier effect is analyzed) three major groups can be defined: marketing channel-focused; farm enterprise-focused; scenario/impact analysis-focused.

Within the first and biggest group, which involves studying the effect of (an increase in) demand in relation to specified marketing channels, most papers (6/13) deal specifically with farmers' markets [53-55] (the latter paper has a dual focus as it also includes an impact analysis; see Group 3) [31,56,57]. The dominance of farmers' markets among the channels that are analyzed is also enhanced by [32]. Other marketing channels are underrepresented; only three papers focus on other specific channels. The authors in [63] study local craft breweries, Mitchell and Lemon [49] addresses an online retailer of local food items, while a local food hub is at the core of [58-60,62], and Watson, Cooke and Kay et al. [61] deal with short food supply chains or local food systems in general. Most of the papers (8/13) apply analytical methods based on input-output (I-O) tables [31,53,54,56,58,60-62], while Mitchell and Lemon [49] provide a simplification of I-O approaches. Three papers draw their conclusions based on an assessment of descriptive statistics [55,59,63], while one paper [57] mostly features qualitative analyses (of interviews and focus group discussions). Eleven studies collect primary research data, and $[60,61]$ apply only secondary data to model sectorial relationships relevant to the local food sector.

In the second group, the impact of organic or non-organic, direct-marketing farm enterprises on their local community is assessed. The following seven papers were identified: $[19,50,64-68]$. Two papers $[64,68]$ out of the seven are based solely on an assessment of descriptive statistics; the rest (3/7) use a further econometric analysis (logit or probit models, or instrumental variable regressions, [65-67]), or an I-O approach $(2 / 7$ papers, $[19,50])$. Two papers are based on published census data or data collected by the USDA $[65,66]$, while the rest also collect primary data.

In the third group, cities or other administrative entities are the focus, and the effect of specific local food-oriented policies is addressed. Five papers were identified [54,69,72] (the latter also analyses the multiplier effect of farmers' markets, so it is classified into both Groups 1 and 3) [70,71]. All the papers use quantitative analytical techniques, three of them are based on I-O models [54,69,70]; one [71] is built on a positive mathematical programming model, and one [72] uses a specific formula for calculations. One paper [54] is solely grounded on primary survey data, and one paper [70] uses a mixed-methods approach, as Impact Analysis for Planning (IMPLAN)-based data are complemented with primary survey data. The rest of the papers rely on multiple published data sources. In terms of localization, all the papers focus on the USA.

\subsection{Method of Analysis}

Another means of classification is the analytical method that is applied. Most of the papers (23/24) use more or less sophisticated quantitative approaches. Three major groups can be identified (i.e., categories into which more than one paper may be included).

The first group consists of 13 papers that apply an input-output approach $[19,31,49,50,53,54,56,58,60-62,69,70])$. Because most studies involve the USA, where this analytical tool is available, the use of IMPLAN, or IMPLAN-based SAM-models is typical (11 papers). Impact Analysis for Planning (IMPLAN) is an economic tool used to quantify the effect of a specific economic activity across the economy. A dataset is arranged in a 546-sector scheme; raw data come from multiple sources such as the Quarterly Census of Employment and Wages, Regional Economic Accounts and other areas; production functions are also provided. As data and the database are editable, the process allows for the creation of various arrangements that depend on the actual research question. The SAM framework was briefly presented above. The work of [56] is derived from the Regional Input-Output Modeling System developed and maintained by the US Bureau of Economic Analysis, while Mitchell and Lemon [49] traced and collected data directly from 
the suppliers of the focal enterprise to produce an estimation of the local multipliers. In terms of the objective of the studies, from the 13 papers, two describe policy impact analyses $[69,70]$, two examine farm enterprises [19,50], and the rest address farmers' markets. The advantages of I-O-related approaches include the fact that data are relatively easy to access, and to operationalize [73]. However, users should modify the default parameters in order to ensure accuracy [32], which often requires subjective judgement [74], and also makes generalization challenging. As Kinnaman [75] notes, because every industry ultimately has a partial responsibility for the spending of any other industry, any I-O approach will estimate more economic activity than actually occurs-a limitation that is rarely discussed.

There are five papers in the second group $[55,59,63,64,68]$. Though they all provide quantitative estimations, they are based on assessments of descriptive statistics that were generated by surveys. Sample selection is subject to bias in all cases; therefore, results are not generalizable and reflect tendencies at most. Three of the papers address different marketing channels, while [64] and [68] focus on farm enterprises.

The third group of papers describe the application of econometric analysis, and all three [65-67] address farm enterprises. Primary and secondary data are also used. According to [32], if the related statistical tests are properly designed, advanced econometrics might be useful for estimating the impact of local food sales on economic variables. Brown, Pender and Wiser et al. [74] propose (in a renewable energy context) that input-output approaches should be used together with econometric analysis to provide valid estimations. Such a mixed approach related to local food systems is generally still forthcoming.

Figure 2 shows the distribution of papers using different methods of analysis.

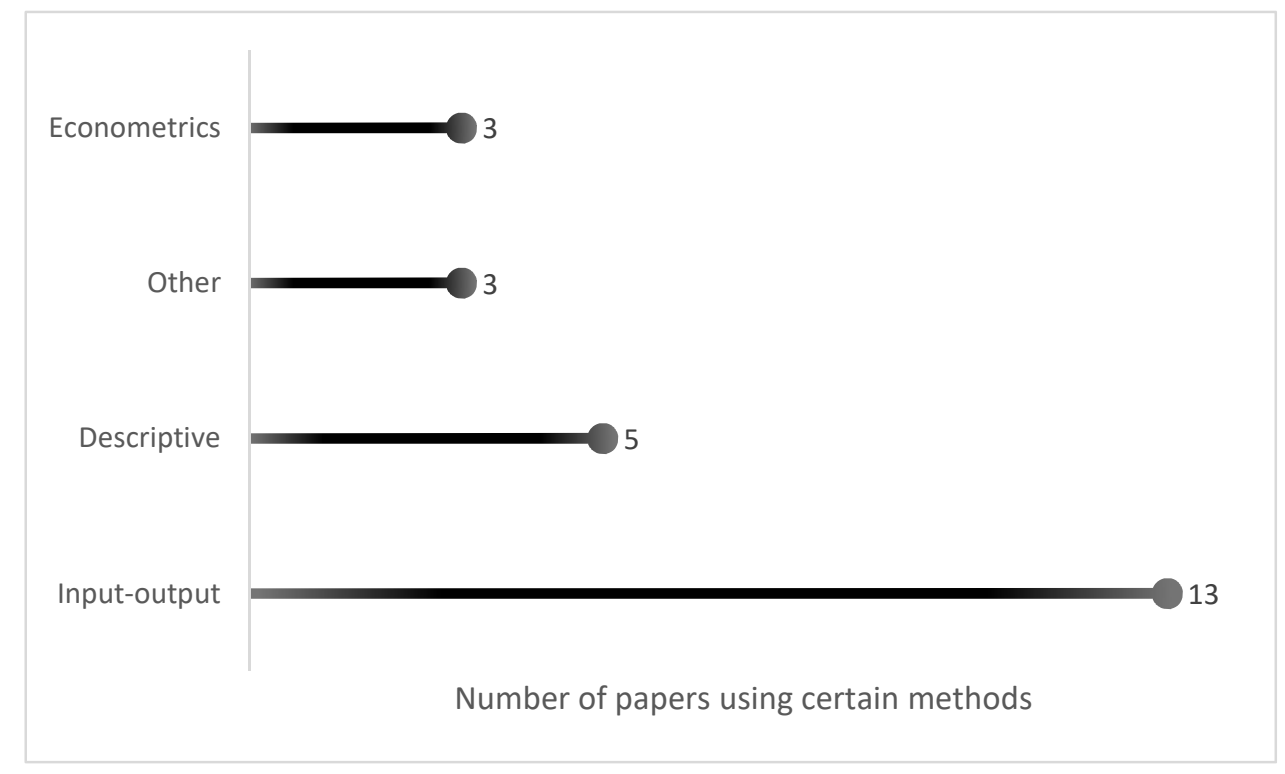

Figure 2. Number of papers that apply different approaches.

To summarize, although input-output models, especially the use of IMPLAN, are the most popular method of analysis, most studies (13/24) are based on other approaches.

\subsection{The Multiplier Effects of Food Relocalization}

The majority of the papers (16/24) conclude unambiguously in favor of the existence of multiplier effects (or find that multiplier effects are greater in the local food context than with conventional agriculture). However, the magnitude and the extent of the former significantly vary. One paper found no impact at all. All four types of multipliers were accounted for. 
Appraisals of the existence of an output multiplier effect $(\mathrm{OM})$ are mixed. OM is verifiably found in 10 cases [31,49,50,53-55,58,60-62]. Rossi, Johnson and Hendrickson [19] and Roberts, Majewski and Sulewski [67] find that the existence of an OM depends on the actual context. According to [57] OMs do not exist in relation to Czech farmers' markets; Schmit, Jablonski and Mansury [70] find that the $\mathrm{OM}$ is lower in the case of the local food sector than for conventional farms, while Gupta and Makov [64], Lambert, Wojan and Sullivan [66] verify that the majority of the spending of local farms went to non-local suppliers, thus no local OM can be calculated. An employment multiplier (EM) effect is claimed to be detectable by ten papers [19,31,50,58-60,62,63,68,69]. Spilková and Perlín [57] find that there is no EM in the Czech context, while Schmit, Jablonski and Mansury [70] find that EMs are lower for local farms compared to conventional ones. An income multiplier effect is found in eight cases [50,58,60,64-66,69,70], while no income multiplier is reported by [57]. Evidence for a value-added multiplier effect (VAM) is also mixed. Six papers report positive VAMs unequivocally [53,56,58,70-72]. Rossi, Johnson and Hendrickson [19] provide clear proof of the context dependence of VAM. Hughes and Isengildina-Massa [54], Spilková and Perlín [57], Brown, Goetz and Ahearn et al. [65] found no VAM.

Seven papers report mixed cases, in the sense that it is claimed that multipliers depend on the context or, in other words, the same multiplier effect is detectable at one location, but not in another $[19,67]$, or that some types of multiplier effects are detectable at one specific location, while others are not $[19,54,64-66,70]$. No relationship can be detected between the findings and the approaches that were applied. Figure 3 displays the number of cases that identified a positive multiplier effect (a) and those where no or a smaller multiplier effect was detected compared to conventional agricultural practices (b), by the type of multiplier.

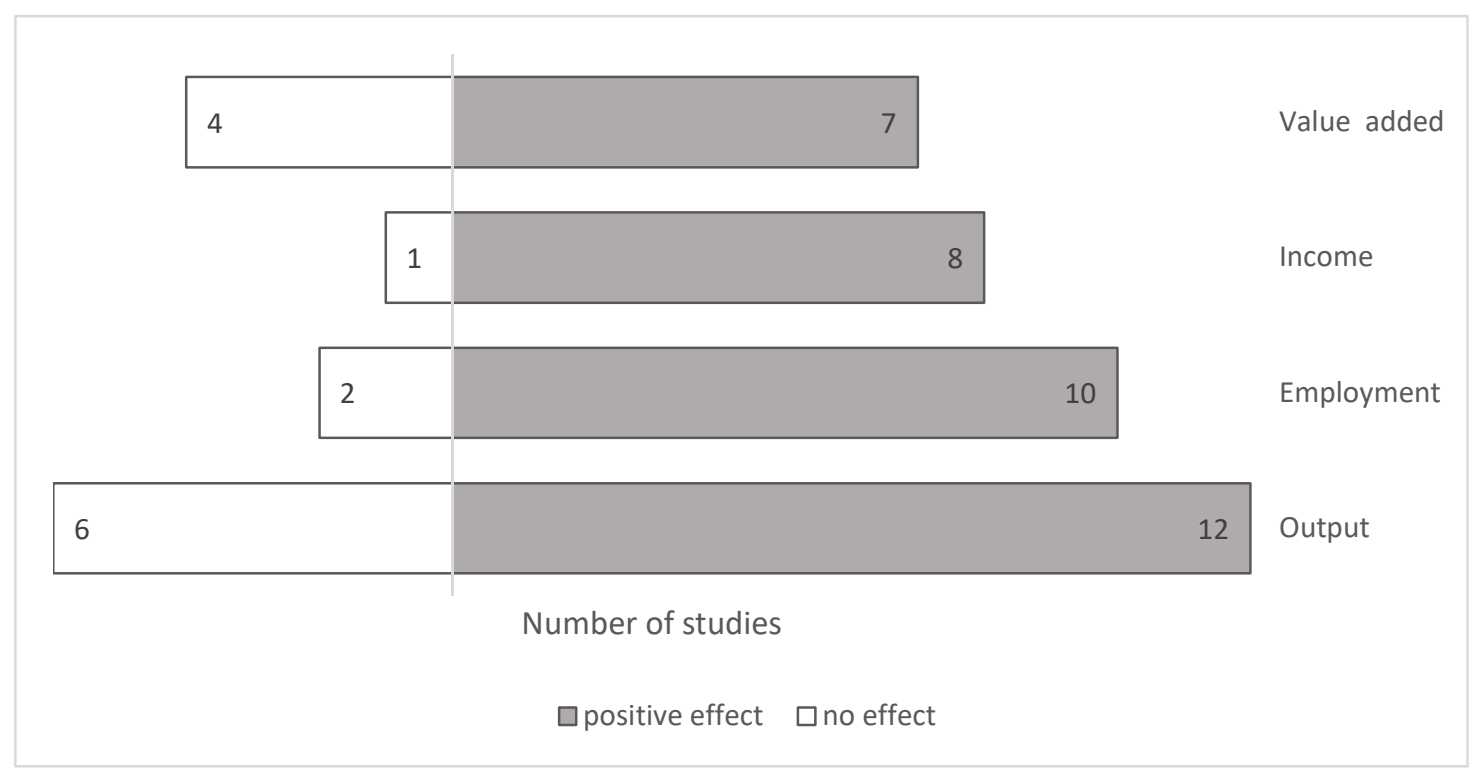

Figure 3. Number of cases in which a specific type of multiplier is addressed. A positive impact was found; no or a lower impact was found compared to conventional practices.

Although direct economic benefits are the focus of this paper, a number of indirect economic benefits are also mentioned in the identified papers (e.g., [55]), such as an increase in the tax base; in the case of farmers' markets, extra profit might be generated through advertising or small businesses incubation; also, additional local employment might be needed on market days, etc. Further, non-marketed benefits may include an increase in trust, etc. These findings are in line with the previous qualitative findings in the literature concerning the benefits of relocalized food $[8,17,76]$.

To conclude, the identification of the presence of at least one type of multiplier effect is highly probable when the impact of relocalized food is considered. Output and employment multipliers are 
most often identified, but the existence of income multipliers is also frequently detected. The occurrence of value-added multipliers seems to be especially context dependent; thus, it is highly questionable whether impacts that are detected that relate to the level of sales or labor translate into an increase in the value added of the local economy.

\subsection{Further Considerations}

The problem of publication bias in the case of systematic reviews and meta-analyses has long been known [77] and should be addressed with special care. Four papers (4/24) were identified that report negative or partly negative results $[54,57,65,67]$. Regarding the relatively high number of such papers (and furthermore, as tendencies were described instead of numerical estimations), publication bias does not seem to have significantly influenced our results.

Although many of the identified studies are associated with limitations, addressing reliability concerns (e.g., the discussion of potential biases) and validity is not typical. Only three papers [31,65,71] deal with both issues. This also means that the key findings of the present review should be regarded with caution.

With respect to the employment multiplier, many of the identified papers (e.g., $[19,50,58,67]$ ) mention that local food systems typically create part-time and seasonal jobs, or operators often have off-farm income sources, unlike with conventional farms or other sectors of the economy. Thus, when jobs are not converted into FTEs, the creative capacity of local food systems is typically an overestimation. Sbicca [78] describes a case in which farm operators rely heavily on voluntary (intern) work; approximately $25 \%$ of all work was paid for. The role of voluntarism has often been stressed in the context of local food systems in general [79], and specifically in relation to certain marketing channels such as farmers' markets [80], urban gardens [81], and vegetable box schemes [82]. Although local food systems might be important in terms of increasing social coherence [83-85], it is challenging to evaluate such benefits in monetary terms; thus, they were omitted from the range of multiplier analyses.

As demonstrated above, the issue of locality is typically raised in a geographical context. However, Gupta and Makov [64] address this topic using an economic approach in terms of whether the inputs of local farms are themselves local. The authors conclude that as the majority of inputs are, in fact, produced globally, as an increase in demand for local food mostly supports non-local suppliers. The idea that the proximity of food production alone does not necessarily equate to local economic development is reflected by [66]. As the actual source of inputs is rarely questioned, it can be assumed that multiplier analyses, especially when outcome or value-added multipliers are studied, provide an overestimation of the related economic contributions.

In terms of the economic impact of local food systems, there is some evidence [86,87] that the emergence of local food systems may be more of a consequence than a driver of rural development, so the potential of the former to enhance local economies is questionable. This issue is not raised by any of the papers in the sample.

\section{Summary and Conclusions}

Food relocalization is an ongoing and increasingly popular trend in wealthier countries $[8,16,17]$. Besides many reported positive effects, such as improved health outcomes, increased social coherence, etc., it is often argued by local food activists that buying more local food directly can foster rural development through multiplier effects [32]. In order to facilitate evidence-based policymaking, this argument was evaluated through a systematic review. Four types of multipliers were analyzed based on [18]: output, employment, income, and value-added multipliers.

Six major databases (ScienceDirect, Wiley Online Library, and Taylor and Francis Online, SpringerLink, AgECON and Emerald) were systematically searched, together with complementary searches performed in Google Scholar. The rigorous process of identification, screening, selection, and inclusion resulted in the identification of 24 peer-reviewed studies published between 2000 and 
2019 for further analysis. The low number of studies permitted a qualitative assessment leading to a number of related statements.

Most importantly, an increase in demand for locally sourced food was found to have a direct positive impact on local economies in $67 \%$ of the identified papers (16/24). For the rest, no impact was detected, or the findings were mixed, with the impact either being context dependent (when different localities were compared), or only one type of multiplier was found to be positive and another type negative (at the same location). The level of agreement is strongest concerning employment and income multipliers. For outcome, and value-added multipliers, the ratio of papers that detected a positive impact to papers that found no impact (or a minimal impact compared to conventional systems) is roughly 2:1, although the total number of papers that find a positive impact is relatively high, at least in the case of the output multiplier (the value-added multiplier is the least frequently studied of the multipliers). This implies that the existence of the latter two types depend more on actual location and context, or that the detected impacts at the level of sales or labor do not necessarily translate into an increase in value added to the local economy. One implication for policymaking is that local food systems-although they might be able to improve quality of life in rural areas, especially when indirect and non-market benefits are also accounted for-may be more appropriately considered as a means of increasing social cohesion than a motor of rural development. This statement is supported by the fact that current analytical methods seem to overestimate the related impacts, and also because the source of inputs used by local producers may not be local due to the complex supply chains of a globalized world. On the other hand, scientific knowledge proves to be limited (especially considering the reliability and validity of existing studies and minimal coverage of countries outside of the USA), so future research is needed before stronger statements can be made.

The further academic contributions of our study concern four areas. Firstly, a framework has been introduced through which the different types of multipliers in the empirical local food context were exhaustively differentiated. Secondly, two methods of classification were proposed in the hope of guiding future research. Based on the focus of the studies, three groups were identified. The first and biggest research area is that of marketing channels. Most studies focus on farmers' markets, while knowledge about other specific channels is sparse. The second group of studies in terms of the object of study involve farm enterprises. Within this group, the number of studies with large, representative samples is currently small; addressing this research gap would increase our understanding of the economic impact of local food systems. The third group of studies focus on scenario/impact analysis. Using another process of classification, the methods of analysis are considered. Though input-output approaches are the most typical of the latter, most studies utilize different methods (such as econometric analyses, or assessments of descriptive statistics). Due to the limitations of the existing models, a combination of input-output tables and advanced econometric methods is proposed for obtaining more reliable estimates about the economic impact of local food systems. Thirdly, our analysis directs the attention of readers to the fact that an additional strand of debate should be introduced when the definition of local food is considered. Until now, the question (as raised by [19]) was whether analyses should focus on specific direct marketing channels or local food systems. In the case of the latter, an additional question emerges related to the definition of "local," which is highly context dependent, both from the perspective of consumers as well as legislation $[17,29,88]$. However, future research about the economic impact of local food should address the issue of locality from an economic point of view, i.e., whether the inputs of production are themselves local; otherwise, effects might be significantly overestimated. Finally, although there is a diversity of applicable methods, the potential for reverse causality regarding whether local food systems are the consequences or drivers of local economic development should be addressed in the future.

Author Contributions: Conceptualization, Z.B. and I.F.; methodology, Z.B. and I.F.; investigation, Z.B., I.F. and V.S.; resources, Z.B. and V.S.; data curation, Z.B. and V.S.; writing-original draft preparation, Z.B.; writing一review and editing, Z.B., I.F. and V.S.; visualization, Z.B. and V.S.; project administration, I.F.; funding acquisition, I.F. All authors have read and agreed to the published version of the manuscript. 
Funding: This research received no external funding.

Acknowledgments: The authors thank the participants of the 174th EAAE Seminar in Matera, Italy, 2019 for views they expressed in relation to the proposed review protocol. Many thanks to Simon Milton for his language-related contributions. Support from the Hungarian National Research, Development and Innovation Fund (grant no. 130485 "The costs and benefits of local foods") is gratefully acknowledged.

Conflicts of Interest: The authors declare no conflict of interest.

\section{References}

1. Furman, C.A.; Papavasiliou, F. Scale and affect in the local food movement. Food Cult. Soc. 2018, 21, $180-195$. [CrossRef]

2. Printezis, I.; Grebitus, C.; Hirsch, S. The price is right!? A meta-regression analysis on willingness to pay for local food. PLoS ONE 2019, 14, e0215847. [CrossRef] [PubMed]

3. Feldmann, C.; Hamm, U. Consumers' perceptions and preferences for local food: A review. Food Qual. Prefer. 2015, 40, 152-164. [CrossRef]

4. Schmitt, E.; Keech, D.; Maye, D.; Barjolle, D.; Kirwan, J. Comparing the sustainability of local and global food chains: A case study of cheese products in Switzerland and the UK. Sustainability 2016, 8, 419. [CrossRef]

5. Janssens, F.; Sezer, C. Marketplaces as an urban development strategy. Built Environment 2013, 39, $169-171$. [CrossRef]

6. Peters, R. Local Food and Short Supply Chains; EU Rural Review: Belgium, Brussels, 2012.

7. Hingley, M.; Boone, J.; Haley, S. Local food marketing as a development opportunity for small UK agri-food businesses. Int. J. Food Syst. Dyn. 2010, 1, 194-203.

8. Martinez, S. Local Food Systems; Concepts, Impacts, and Issues; Diane Publishing: Collingdale, PA, USA, 2010.

9. Mount, P. Growing local food: Scale and local food systems governance. Agric. Hum. Values 2012, 29, 107-121. [CrossRef]

10. Scharber, H.; Dancs, A. Do locavores have a dilemma? Economic discourse and the local food critique. Agric. Hum. Values 2016, 33, 121-133. [CrossRef]

11. Ilbery, B.; Watts, D.; Simpson, S.; Gilg, A.; Little, J. Mapping local foods: Evidence from two English regions. Br. Food J. 2006, 108, 213-225. [CrossRef]

12. Renting, H.; Marsden, T.K.; Banks, J. Understanding alternative food networks: Exploring the role of short food supply chains in rural development. Environ. Plan. A 2003, 35, 393-411. [CrossRef]

13. Willis, D.B.; Carpio, C.E.; Boys, K.A. Supporting local food system development through food price premium donations: A policy proposal. J. Agric. Appl. Econ. 2016, 48, 192-217. [CrossRef]

14. DuPuis, E.M.; Goodman, D. Should we go "home" to eat? toward a reflexive politics of localism. J. Rural. Stud. 2005, 21, 359-371. [CrossRef]

15. Goodman, D. Rural Europe redux? Reflections on alternative agro-food networks and paradigm change. Sociol. Rural. 2004, 44, 3-16. [CrossRef]

16. Deller, S.C.; Lamie, D.; Stickel, M. Local foods systems and community economic development. Community Dev. 2017, 48, 612-638. [CrossRef]

17. Kneafsey, M.; Venn, L.; Schmutz, U.; Balázs, B.; Trenchard, L.; Eyden-Wood, T.; Bos, E.; Sutton, G.; Blackett, M. Short food supply chains and local food systems in the EU. A state of play of their socio-economic characteristics. JRC Sci. Policy Rep. 2013, 123, 129.

18. Miller, R.E.; Blair, P.D. Input-output Analysis: Foundations and Extensions; Cambridge University Press: Cambridge, UK, 2009.

19. Rossi, J.D.; Johnson, T.G.; Hendrickson, M. The economic impacts of local and conventional food sales. J. Agric. Appl. Econ. 2017, 49, 555-570. [CrossRef]

20. Domański, B.; Gwosdz, K. Multiplier effects in local and regional development. Quaest. Geogr. 2010, 29, 27-37. [CrossRef]

21. D’Hernoncourt, J.; Cordier, M.; Hadley, D. Input-output multipliers specification sheet and supporting material. Available online: http://www.coastal-saf.eu/output-step/pdf/Specification\%20sheet\%20I_O_final. pdf (accessed on 24 April 2020).

22. Hughes, D.W. Policy uses of economic multiplier and impact analysis. Choices 2003, 18, 25-30. 
23. Van Wyk, L.; Saayman, M.; Rossouw, R.; Saayman, A. Regional economic impacts of events: A comparison of methods. S. Afr. J. Econ. Manag. Sci. 2015, 18, 155-176. [CrossRef]

24. Hartono, D.; Nugroho, A. Impacts of Food Prices on the Economy: Social Accounting Matrix and Microsimulation Approach In Indonesia. Rev. Urban Reg. Dev. Stud. 2019, 31, 137-154.

25. Mansury, Y.; Hara, T. Impact of organic food agritourism on a small rural economy: A social accounting matrix approach. SSRN Electron. J. 2006. [CrossRef]

26. Partridge, M.D.; Rickman, D.S. Computable general equilibrium (CGE) modelling for regional economic development analysis. Reg. Stud. 2010, 44, 1311-1328. [CrossRef]

27. West, G.R. Comparison of input-output, input-output+ econometric and computable general equilibrium impact models at the regional level. Econ. Syst. Res. 1995, 7, 209-227. [CrossRef]

28. Gelan, A. Cash or food aid? A general equilibrium analysis for Ethiopia. Dev. Policy Rev. 2006, 24, 601-624. [CrossRef]

29. Pirog, R.; Rasmussen, R. Food, fuel and the future: Consumer perceptions of local food, food safety and climate change in the context of rising prices. Available online: https://www.leopold.iastate.edu/files/pubs-and-papers/2008-09-food-fuel-and-future-consumerperceptions-local-food-food-safety-and-climate-change-context-rising-p.pdf (accessed on 24 April 2020).

30. Brown, C.; Miller, S. The impacts of local markets: A review of research on farmers markets and community supported agriculture (CSA). m. J. Agric. Econ. 2008, 90, 1298-1302. [CrossRef]

31. Hughes, D.W.; Brown, C.; Miller, S.; McConnell, T. Evaluating the economic impact of farmers' markets using an opportunity cost framework. J. Agric. Appl. Econ. 2008, 40, 253-265. [CrossRef]

32. O’Hara, J.K.; Pirog, R. Economic impacts of local food systems: Future research priorities. J. Agric. Food Syst. Community Dev. 2013, 3, 35-42. [CrossRef]

33. Chiffoleau, Y.; Millet-Amrani, S.; Rossi, A.; Rivera-Ferre, M.G.; Merino, P.L. The participatory construction of new economic models in short food supply chains. J. Rural. Stud. 2019, 68, 182-190. [CrossRef]

34. Berti, G.; Mulligan, C. Competitiveness of small farms and innovative food supply chains: The role of food hubs in creating sustainable regional and local food systems. Sustainability 2016, 8, 616. [CrossRef]

35. Hendrickson, M.K.; Heffernan, W.D. Opening spaces through relocalization: Locating potential resistance in the weaknesses of the global food system. Sociol. Rural. 2002, 42, 347-369. [CrossRef]

36. Lerner, A.M.; Eakin, H. An obsolete dichotomy? Rethinking the rural-urban interface in terms of food security and production in the global south. Geogr. J. 2011, 177, 311-320. [CrossRef] [PubMed]

37. Olsson, J. Rural-urban spatial interaction in the global south: Long-distance mobility changes, desires and restrictions over two decades in rural philippines. Geogr. Ann. Ser. B Hum. Geogr. 2012, 94, 287-304. [CrossRef]

38. Warshawsky, D.N. Civil society and urban food insecurity: Analyzing the roles of local food organizations in Johannesburg. Urban Geogr. 2014, 35, 109-132. [CrossRef]

39. Mulrow, C.D. Systematic reviews: Rationale for systematic reviews. BMJ 1994, 309, 597-599. [CrossRef]

40. Moher, D.; Liberati, A.; Tetzlaff, J.; Altman, D.G. Preferred reporting items for systematic reviews and meta-analyses: The PRISMA statement. Ann. Intern. Med. 2009, 151, 264-269. [CrossRef]

41. Boaz, A.; Ashby, D.; Young, K. Systematic reviews: What have they got to offer evidence based policy and practice? Available online: https://www.kcl.ac.uk/sspp/departments/politicaleconomy/research/cep/pubs/ papers/assets/wp2.pdf (accessed on 24 April 2020).

42. Garside, R. Should we appraise the quality of qualitative research reports for systematic reviews, and if so, how? Innov. Eur. J. Soc. Sci. Res. 2014, 27, 67-79. [CrossRef]

43. Beller, E.M.; Glasziou, P.P.; Altman, D.G.; Hopewell, S.; Bastian, H.; Chalmers, I.; Gøtzsche, P.C.; Lasserson, T.; Tovey, D.; Group, P.f.A. PRISMA for abstracts: Reporting systematic reviews in journal and conference abstracts. PLoS Med. 2013, 10, e1001419. [CrossRef]

44. Green, R.; Cornelsen, L.; Dangour, A.D.; Turner, R.; Shankar, B.; Mazzocchi, M.; Smith, R.D. The effect of rising food prices on food consumption: Systematic review with meta-regression. BMJ 2013, 346, f3703. [CrossRef]

45. Kataike, J.; Gellynck, X. 22 Years of governance structures and performance: What has been achieved in agrifood chains and beyond? A review. Agriculture 2018, 8, 51. [CrossRef]

46. Poulsen, M.N.; McNab, P.R.; Clayton, M.L.; Neff, R.A. A systematic review of urban agriculture and food security impacts in low-income countries. Food Policy 2015, 55, 131-146. [CrossRef] 
47. Dias, C.S.; Rodrigues, R.G.; Ferreira, J.J. Agricultural entrepreneurship: Going back to the basics. J. Rural. Stud. 2019, 70, 125-138. [CrossRef]

48. Freedman, D.A.; Vaudrin, N.; Schneider, C.; Trapl, E.; Ohri-Vachaspati, P.; Taggart, M.; Cascio, M.A.; Walsh, C.; Flocke, S. Systematic review of factors influencing farmers' market use overall and among low-income populations. J. Acad. Nutr. Diet. 2016, 116, 1136-1155. [CrossRef] [PubMed]

49. Mitchell, A.; Lemon, M. Using the LM3 method to evaluate economic impacts of an on-line retailer of local food in an English market town. Local Econ. 2019, 34, 51-67. [CrossRef]

50. Pesch, R.; Tuck, B. Developing a production function for small-scale farm operations in Central Minnesota. J. Agric. Food Syst. Community Dev. 2019, 8, 27-36. [CrossRef]

51. Vieira, L.C.; Serrao-Neumann, S.; Howes, M.; Mackey, B. Unpacking components of sustainable and resilient urban food systems. J. Clean. Prod. 2018, 200, 318-330. [CrossRef]

52. Pitt, E.; Gallegos, D.; Comans, T.; Cameron, C.; Thornton, L. Exploring the influence of local food environments on food behaviours: A systematic review of qualitative literature. Public Health Nutr. 2017, 20, 2393-2405. [CrossRef]

53. Henneberry, S.R.; Whitacre, B.E.; Agustini, H.N. An evaluation of the economic impacts of Oklahoma farmers markets. J. Food Distrib. Res. 2009, 40, 64-78.

54. Hughes, D.W.; Isengildina-Massa, O. The economic impact of farmers' markets and a state level locally grown campaign. Food Policy 2015, 54, 78-84. [CrossRef]

55. Bubinas, K. Farmers markets in the post-industrial city. City Soc. 2011, 23, 154-172. [CrossRef]

56. Sadler, R.C.; Clark, M.A.R.; Gilliland, J.A. An economic impact comparative analysis of farmers' markets in Michigan and Ontario. J. Agric. Food Syst. Community Dev. 2013, 3, 61-81. [CrossRef]

57. Spilková, J.; Perlín, R. Farmers' markets in Czechia: Risks and possibilities. J. Rural. Stud. 2013, 32, $220-229$. [CrossRef]

58. Hodges, A.W.; Stevens, T.J.; Wysocki, A.F. Local and regional food systems in Florida: Values and economic impacts. J. Agric. Appl. Econ. 2014, 46, 285-298. [CrossRef]

59. Mundler, P.; Laughrea, S. The contributions of short food supply chains to territorial development: A study of three Quebec territories. J. Rural. Stud. 2016, 45, 218-229. [CrossRef]

60. Miller, S.R.; Mann, J.; Barry, J.; Kalchik, T.; Pirog, R.; Hamm, M.W. A replicable model for valuing local food systems. J. Agric. Appl. Econ. 2015, 47, 441-461. [CrossRef]

61. Watson, P.; Cooke, S.; Kay, D.; Alward, G.; Morales, A. A method for evaluating the economic contribution of a local food system. J. Agric. Resour. Econ. 2017, 42, 180-194.

62. Jablonski, B.B.; Schmit, T.M.; Kay, D. Assessing the economic impacts of food hubs on regional economies: A framework that includes opportunity cost. Agric. Resour. Econ. Rev. 2016, 45, 143-172. [CrossRef]

63. Argent, N. Heading down to the local? Australian rural development and the evolving spatiality of the craft beer sector. J. Rural. Stud. 2018, 61, 84-99. [CrossRef]

64. Gupta, C.; Makov, T. How global is my local milk? Evaluating the first-order inputs of "local" milk in Hawai 'i. Agric. Hum. Values 2017, 34, 619-630. [CrossRef]

65. Brown, J.P.; Goetz, S.J.; Ahearn, M.C.; Liang, C.-1. Linkages between community-focused agriculture, farm sales, and regional growth. Agric. Hum. Values 2014, 28, 5-16. [CrossRef]

66. Lambert, D.; Wojan, T.; Sullivan, P. Farm business and household expenditure patterns and local communities: Evidence from a national farm survey. Rev. Agric. Econ. 2009, 31, 604-626. [CrossRef]

67. Roberts, D.; Majewski, E.; Sulewski, P. Farm household interactions with local economies: A comparison of two EU case study areas. Land Use Policy 2013, 31, 156-165. [CrossRef]

68. Lobley, M.; Butler, A.; Reed, M. The contribution of organic farming to rural development: An exploration of the socio-economic linkages of organic and non-organic farms in England. Land Use Policy 2009, 26, 723-735. [CrossRef]

69. Conner, D.S.; Knudson, W.A.; Hamm, M.W.; Peterson, H.C. The food system as an economic driver: Strategies and applications for Michigan. J. Hunger. Environ. Nutr. 2008, 3, 371-383. [CrossRef]

70. Schmit, T.M.; Jablonski, B.B.; Mansury, Y. Assessing the economic impacts of local food system producers by scale: A case study from New York. Econ. Dev. Q. 2016, 30, 316-328. [CrossRef]

71. Khan, S.S.; Arita, S.; Howitt, R.; Leung, P. A calibrated model of local food system of Hawaii: What are the economic implications of the state's food goals and policies? Nat. Resour. Model. 2018, 32, e12196. [CrossRef]

72. Grewal, S.S.; Grewal, P.S. Can cities become self-reliant in food? Cities 2012, 29, 1-11. [CrossRef] 
73. Miller, S.R.; Mann, J.T. On Measuring the Importance of Local Food to Regional Economies; Mid-Contenting Regional Science Association: Charlotte, NC, USA, 2016.

74. Brown, J.P.; Pender, J.; Wiser, R.; Lantz, E.; Hoen, B. Ex post analysis of economic impacts from wind power development in US counties. Energy Econ. 2012, 34, 1743-1754. [CrossRef]

75. Kinnaman, T.C. The economic impact of shale gas extraction: A review of existing studies. Ecol. Econ. 2011, 70, 1243-1249. [CrossRef]

76. Gerbasi, G.T. Athens farmers' market: Evolving dynamics and hidden benefits to a southeast ohio rural community. FOCUS Geogr. 2006, 49, 1. [CrossRef]

77. Thornton, A.; Lee, P. Publication bias in meta-analysis: Its causes and consequences. J. Clin. Epidemiol. 2000, 53, 207-216. [CrossRef]

78. Sbicca, J. Food labor, economic inequality, and the imperfect politics of process in the alternative food movement. Agric. Hum. Values 2015, 32, 675-687. [CrossRef]

79. Balázs, B. Local Food System Development in Hungary. Int. J. Sociol. Agric. Food 2012, 19, 403-421.

80. Beckie, M.A.; Kennedy, E.H.; Wittman, H. Scaling up alternative food networks: Farmers' markets and the role of clustering in western Canada. Agric. Hum. Values 2012, 29, 333-345. [CrossRef]

81. Burdine, J.D.; Taylor, D.E. Neighbourhood characteristics and urban gardens in the Toledo metropolitan area: Staffing and voluntarism, food production, infrastructure, and sustainability practices. Local Environ. 2018, 23, 198-219. [CrossRef]

82. Szabó, I.; Lehota, J.; Magda, R. Purchase of Fresh Fruits and Vegetables Through Box Schemes in Hungary-Opportunities and Hindering Factors on the Way to Sustainability. Visegrad J. Bioeconomy Sustain. Dev. 2019, 8, 37-41. [CrossRef]

83. Hedberg II, R.C.; Zimmerer, K.S. What's the market got to do with it? Social-ecological embeddedness and environmental practices in a local food system initiative. Geoforum 2020, 110, 35-45. [CrossRef]

84. Nousiainen, M.; Pylkkänen, P.; Saunders, F.; Seppänen, L.; Vesala, K.M. Are alternative food systems socially sustainable? A case study from Finland. J. Sustain. Agric. 2009, 33, 566-594. [CrossRef]

85. Sage, C. Social embeddedness and relations of regard: Alternative 'good food'networks in south-west Ireland. J. Rural. Stud. 2003, 19, 47-60. [CrossRef]

86. Ricketts Hein, J.; Ilbery, B.; Kneafsey, M. Distribution of local food activity in England and Wales: An index of food relocalization. Reg. Stud. 2006, 40, 289-301. [CrossRef]

87. Tregear, A. Progressing knowledge in alternative and local food networks: Critical reflections and a research agenda. J. Rural. Stud. 2011, 27, 419-430. [CrossRef]

88. Benedek, Z.; Fertő, I.; Molnár, A. Off to market: But which one? Understanding the participation of small-scale farmers in short food supply chains-A Hungarian case study. Agric. Hum. Values 2018, 35, 383-398. [CrossRef]

(C) 2020 by the authors. Licensee MDPI, Basel, Switzerland. This article is an open access article distributed under the terms and conditions of the Creative Commons Attribution (CC BY) license (http://creativecommons.org/licenses/by/4.0/). 